\title{
Recycling of Polyethylene Terephthalate Using High-pressure Steam Treatment
}

\author{
By Ai NORITAKE, ${ }^{1}$ Mitsuo HorI, ${ }^{2}$ Mikiji ShIGEMATSU, ${ }^{3}$ and Mitsuhiko TANAHASHI ${ }^{3, *}$
}

In this study, polyethylene terephthalate (PET) was broken into fine particles during high-pressure steam treatment without crushing or using acidic or alkaline catalysts and solvents. Scanning electron microscopy (SEM) images showed that PET was decomposed from the surface by the high-pressure steam. ${ }^{1} \mathrm{H}$ NMR and $\mathrm{CP} / \mathrm{MAS}{ }^{13} \mathrm{C}$ NMR spectra revealed that the molecular weight of PET gradually decreased to that of an oligomer with increasing the high-pressure steam treatment time, and then reacted to a monomer. Differential scanning calorimetry (DSC) showed a decrease in the glass transition temperature $\left(T_{\mathrm{g}}\right)$, crystallization temperature $\left(T_{\mathrm{c}}\right)$, and melting temperature $\left(T_{\mathrm{m}}\right)$. X-Ray diffraction (XRD) measurements indicated that crystallinity increased with high-pressure steam treatment time. Decomposed PET consisting of hydroxyl groups and carboxylic acid thus repolymerize into new products. This investigation showed that a PET recycling method can be established easily, which had no adverse effect on the environment.

KEY WORDS: Polyethylene Terephtalate / Recycle / High-pressure Steam / Decomposition /

Polyethylene terephthalate (PET) is commonly used to package soft drinks, food items, cosmetics, and household cleaners because of its high strength, thermo stability, transparency, low weight and shatter resistance. Therefore, global PET resin consumption is increasing every year. In Japan, the amount of recovered PET bottles has increased annually after the enforcement of the law for the promotion of sorted collection and recycling of containers and packaging in 1997. 1,2 There is growing concern about environmental issues and this has led to increase in studies on the recycling of waste plastic. Currently, various methods exist for recycling PET wastes, such as material recycling by heat press and injection molding. These methods are applied to textile filaments and sheets. Moreover, chemical treatments such as glycol decomposition, ${ }^{3,4}$ amine decomposition, ${ }^{5}$ and supercritical methanol ${ }^{6-9}$ or water decomposition ${ }^{10,11}$ are also well known. However, the recycling of PET bottles involves size reduction by shredding, sorting, grinding, hot water washing, drying, melting, extruding, chopping into pellets, hot air drying, and molding into recycled products. During material recycling, there is substantial degradation of molecular structure, resulting in decreased viscosity and mechanical strength of the molten material, while chemical treatment requires the use of expensive catalysts, solvents, and equipment. For industrial utilization, it requires additional secondary treatment of decomposed products, which is an additional cost factor. Recently, considerable attention has been focused on the supercritical methanol or water decomposition of PET but the reaction speed is very fast. Also the equipment required is very expensive, and the treatable amount is limited.

We have proposed a unique PET recycling method using high-pressure steam treatment, which is milder than the supercritical reaction. Smashed PET can be obtained easily by this method without crushing and without acidic or alkaline catalysts and solvents. The method saves time and money, and thus is important for PET recycling, and environment friendly.

\section{EXPERIMENTAL}

\section{Materials}

The samples, empty PET bottles used for the packaging of soft drinks, were cut into pieces of approximately $30 \mathrm{~mm} \times 80 \mathrm{~mm}$.

\section{Decomposition of PET by High-pressure Steam Treatment}

The samples were treated in a high-pressure steam apparatus HTP 40/60, manufactured by Hisaka Works, Ltd., Figure 1. Inner volume was approximately $0.08 \mathrm{~m}^{3}$, and maximum working pressure of the reactor was $2.0 \mathrm{MPa}$. The device was set to $1.6 \mathrm{MPa}\left(200^{\circ} \mathrm{C}\right)$ by introducing steam. PET samples

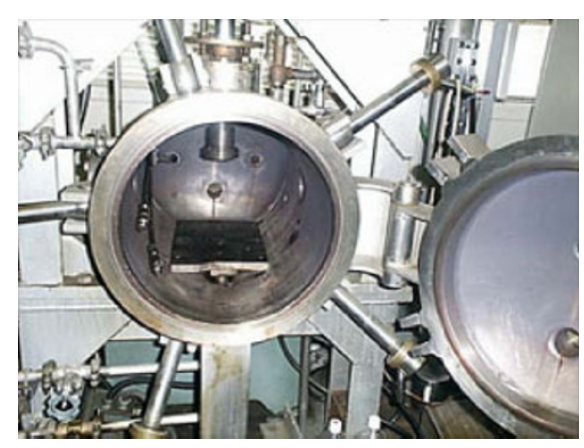

Figure 1. High-pressure steam treatment apparatus.

\footnotetext{
${ }^{1}$ Environment and Renewable Energy Systems, Graduate School of Engineering, Gifu University, 1-1 Yanagido, Gifu 501-1193, Japan

${ }^{2}$ Aquastream Corporation, 627 Tsugiki, Gifu 501-6122, Japan

${ }^{3}$ Division of Utilization of Biological Resources, Faculty of Applied Biological Sciences, Gifu University, 1-1 Yanagido, Gifu 501-1193, Japan

*To whom correspondence should be addressed (Tel: +81-58-293-2922, Fax: +81-58-293-2922, E-mail: dxv3688@cc.gifu-u.ac.jp).
} 
were subjected to high-pressure steam treatment for 30,60 , 120,180 , and $240 \mathrm{~min}$ and then ground into fine powder with a pestle and mortar for analysis without scanning electron microscopy (SEM) observations.

\section{Analysis of Decomposed PET}

The treated sample surface was observed by SEM using S-3000N, manufactured by Hitachi, Ltd. All the surfaces were coated with a thin layer of osmium prior to SEM observation.

Thermal behavior and X-ray diffraction (XRD) patterns were examined, and the characteristics of the decomposed PET were observed. A differential scanning calorimetry (DSC) device EXSTAR-6000 equipped with a mechanical intracooler under nitrogen purge, manufactured by SII NanoTechnology Inc., was used to study thermal behavior. The treated samples were placed in open aluminium pans, and features were recorded at a heating rate of $5^{\circ} \mathrm{C} \mathrm{min}^{-1}$. The samples were initially heated to $200{ }^{\circ} \mathrm{C}$ and then quenched to $25^{\circ} \mathrm{C}$, followed by a second heating to $300^{\circ} \mathrm{C}$. To provide the same thermal history for all samples, data for the second heating scan were used in DSC.

XRD patterns of the treated samples were examined with a Rigaku RINT-Ultima III/PC using $\mathrm{Cu} \mathrm{K} \alpha$ radiation at a small glazing angle $\left(5^{\circ}\right) 2 \theta$ scan.

$\mathrm{CP} / \mathrm{MAS}{ }^{13} \mathrm{C} \mathrm{NMR}$ spectra of the treated samples were measured to study changes in the structures of the decomposed PET, using a JEOL JNM-ECA 500 spectrometer with an NM93030 CPM probe. The samples were placed in a cylindrical rotor made of zirconia and spun at $1.2 \mathrm{kHz}$. Contact and repetition times were $2 \mathrm{~ms}$ and $5 \mathrm{~s}$, respectively.

To determine molecular weight, decomposed PET was examined by gel permeation chromatography (GPC), using a Shodex GPC System-21 equipped with UV detector. A chloroform/1,1,1,3,3,3-hexafluoro-2-propanol(HFIP) (98/2 vol \%) mixture was used as the eluent that was passed through 3 series of columns, i.e., two of TSK gel GMH $\mathrm{XL}_{\mathrm{XL}}$ and one of TSK gel $\mathrm{G} 2000 \mathrm{H}_{\mathrm{XL}}$ (Tosoh Corp.) at a flow rate of $0.7 \mathrm{~mL} / \mathrm{min}$. Column temperature was $40^{\circ} \mathrm{C}$.

The degree of decomposition of PET was confirmed based on number average molecular weight, and number of hydroxyls and acids calculated by ${ }^{1} \mathrm{H}$ NMR. The ${ }^{1} \mathrm{H}$ NMR spectra of the treated samples were measured with AVANCE 500, manufactured by Bruker Japan Co., Ltd. Equal volums of chloroform-d $\left(\mathrm{CDCl}_{3}\right)$ and HFIP were mixed and $0.3 \mathrm{~mL}$ of the mixture was used to dissolve each sample. $30 \mu \mathrm{l}$ pyridine-d and $0.3 \mathrm{~mL}$ $\mathrm{CDCl}_{3}$ were mixed together to determine the fixed number of hydroxyls. For determination of the fixed number of acids, $0.3 \mathrm{~mL} \mathrm{CDCl}_{3}$ and HFIP mixture to dissolve each sample and after that $0.3 \mathrm{~mL} \mathrm{CDCl}_{3}$ and $60 \mu \mathrm{l}$ triethylamine $\left(0.2 \mathrm{~mol} \mathrm{~L}^{-1}\right.$ in $\mathrm{CDCl}_{3}$ ) were added. Samples that could not completely melt after 120-, 180-, and 240-min treatment were subjected to centrifugation sediment analysis by ${ }^{1} \mathrm{H}$ NMR with dimethyl sulfoxide-d. Sediment was weighed after drying, and the ratio of solid to molten parts was calculated.

To analyze vapor and liquid after 240-min treatment, the samples were cooled to room temperature. The collected liquid sample was then mixed with ethylenediamine of $0.3 \mathrm{ML}^{-1}$ and analyzed by ${ }^{1} \mathrm{H}$ NMR with $\mathrm{D}_{2} \mathrm{O}$. The $0.3 \mathrm{ML}^{-1}$ ethylenediamine solution was served as the standard for determining and comparing integral ${ }^{1} \mathrm{H}$ NMR. The weight of the ethylene glycol (EG) was thus determined from integration of the ${ }^{1} \mathrm{H}$ NMR value.

\section{RESULTS AND DISCUSSION}

The samples were gradually broken into small pieces during the high-pressure steam treatment, and the samples treated for more than $120 \mathrm{~min}$ ground into fine white particles. SEM images of the surface are shown in Figure 2. The surface of the samples decomposed due to the high-pressure steam. Because steam at a high temperature and pressure is highly ionized into $\mathrm{H}^{+}$and $\mathrm{OH}^{-}\left(\log \mathrm{Kw} \fallingdotseq-11\right.$ at $\left.200{ }^{\circ} \mathrm{C}\right)$, the hydrolysis of ester bonds in PET is accelerated due to the high-pressure steam attack on the surface. ${ }^{12,13}$

The results of the thermal behavior of the samples evaluated by DSC are shown in Figure 3. From Figure 3 the glass transition temperature $\left(T_{\mathrm{g}}\right)\left(72^{\circ} \mathrm{C}-66^{\circ} \mathrm{C}\right)$, crystallization temperature $\left(T_{\mathrm{c}}\right)\left(126^{\circ} \mathrm{C}-97^{\circ} \mathrm{C}\right)$, and melting temperature $\left(T_{\mathrm{m}}\right)$ $\left(223^{\circ} \mathrm{C}-182^{\circ} \mathrm{C}\right)$, decreased with high-pressure steam treatment time below $120 \mathrm{~min}$. Expansion of the endothermic peak of

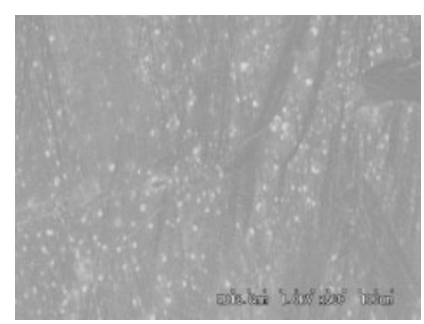

$0 \min$

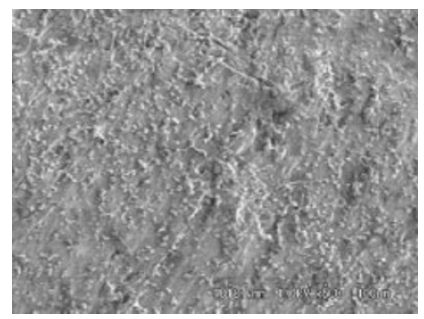

$60 \mathrm{~min}$

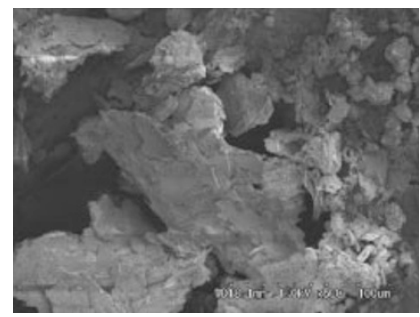

$180 \mathrm{~min}$

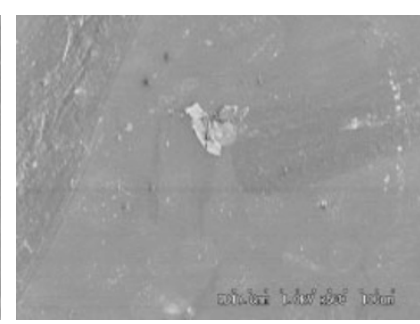

$30 \mathrm{~min}$

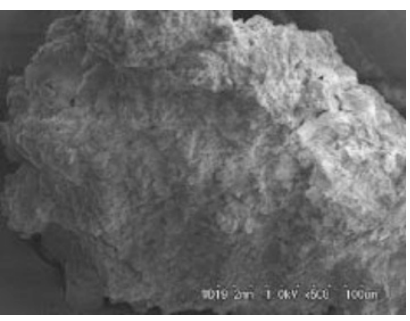

$120 \mathrm{~min}$

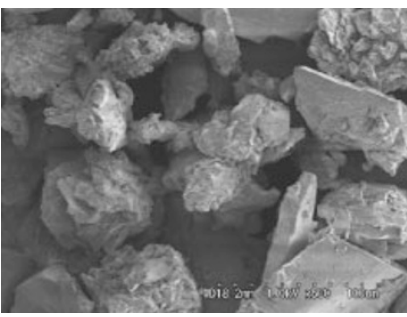

$240 \mathrm{~min}$
Figure 2. Variation of surface of treated PET with high-pressure steam treatment time. 


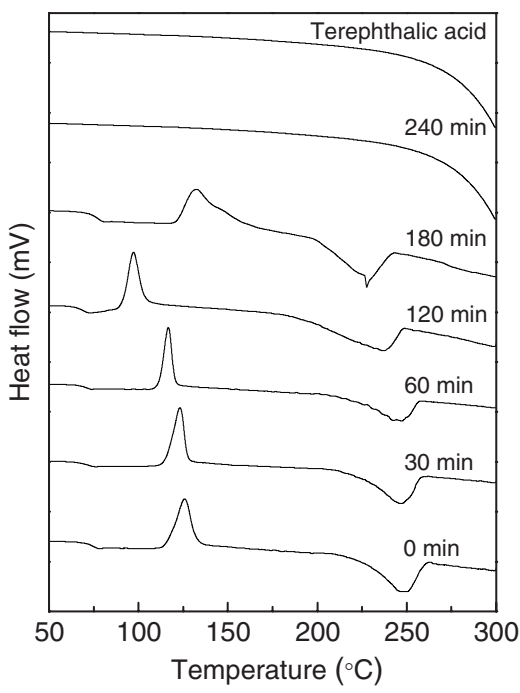

Figure 3. Variation in DSC curves of PET with high-pressure steam treatment time.

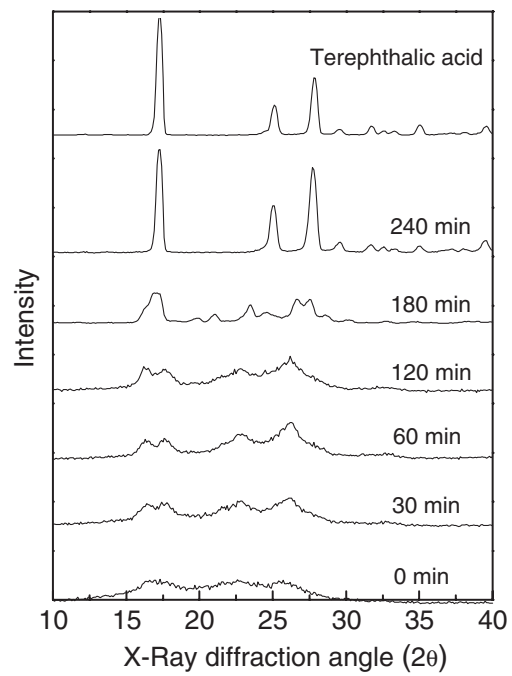

Figure 4. Variation in X-ray diffraction patterns of PET with high-pressure steam treatment time.

melting of decomposed PET with increase of high-pressure steam treatment time indicates a widening of molecular weight. The curve obtained during the 60-min high-pressure steam treatment shows that the crystallization signal was sharpest due to further crystallization. However, when reaction time was increased to 180 and $240 \mathrm{~min}$, the decomposition reached an advanced stage, so that $T_{\mathrm{g}}, T_{\mathrm{c}}$, and $T_{\mathrm{m}}$ completely changed phases. High-pressure steam treatment time of $240 \mathrm{~min}$, no peaks of the DSC curve were detected since the sublimation pattern of the sample was the same as that of the terephthalic acid (TPA) curve. XRD studies initially showed minor crystallization of the raw material and then the rate of crystallization increased with steam treatment time (Figure 4). DSC curves and XRD patterns showed progressive decrease in molecular weight until TPA with increasing high-pressure steam treatment time.
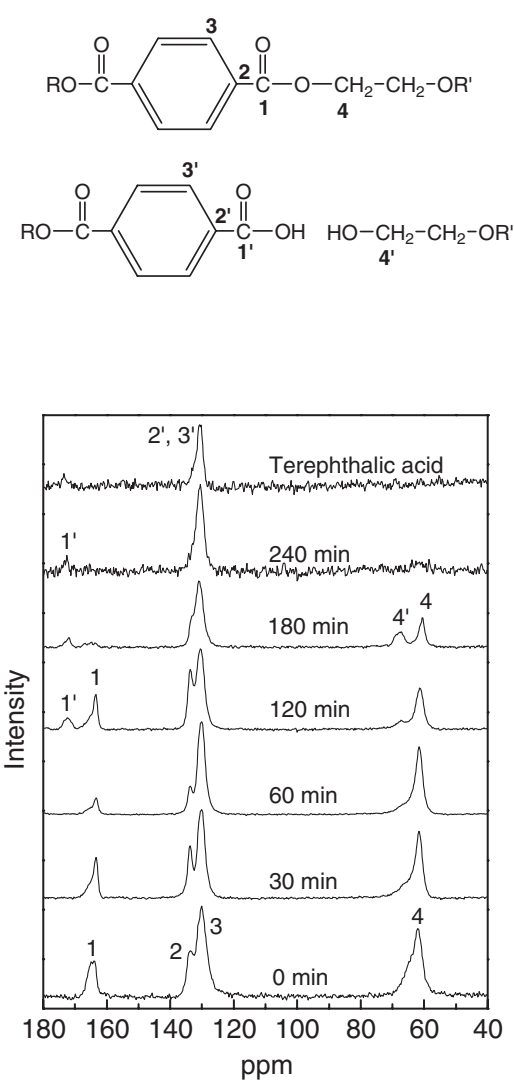

Figure 5. Variation in $\mathrm{CP} / \mathrm{MAS}{ }^{13} \mathrm{C}$ NMR spectra of PET with high-pressure steam treatment time.

Figure 5 shows variation in the $\mathrm{CP} / \mathrm{MAS}{ }^{13} \mathrm{C}$ NMR spectra of the treated samples and changes in the molecular structure. The sample treated for $120 \mathrm{~min}$ had the following peaks: $\delta 172$ $(\mathrm{COOH}), 163(\mathrm{COO}), 134$ (aromatic $C$ adjacent to the ester), 133 (aromatic $C), 67\left(\mathrm{HOCH}_{2}\right)$, and $61\left(\mathrm{COOCH}_{2}\right)$. The appearance of the signal based on the carbonyl carbon in the carboxylic acid (172 ppm) of the spectra of the samples treated for $120 \mathrm{~min}$ indicates increase in the rate of hydrolysis of the ester. Signals based on the ethylene carbons (61 ppm) decreased gradually with the steam treatment time. At $240 \mathrm{~min}$, the signal based on the unprotonated carbonyl carbon in the carboxylic acid (172 ppm) was weak, and thus had very little signal due to the nuclear Overhauser effect. The signal of the ethylene carbon adjacent to the hydroxyl (67 ppm) disappeared due to conversion of EG into its monomer, which was then removed from the solid. In Figure 5, when C2 (134 ppm) shifts to a high magnetic field and C3 (130 ppm) is at a low magnetic field, the carboxylic esters hydrolyze to carboxylic acid, and the peaks of C2 and C3 overlap when completely decomposed to the monomer (131 ppm).

Results of GPC analysis of PET after 0 and 30 min treatment are shown in Table I. The number average $\left(M_{\mathrm{n}}\right)$ and weight average molecular weights $\left(M_{\mathrm{w}}\right)$ decrease and the molecular weight distribution increases with the corresponding increase in the treatment period. GPC could not be performed when the samples were treated for more than $60 \mathrm{~min}$ because of the 
Table I. Properties of the sample measured by GPC

\begin{tabular}{cccc}
\hline $\begin{array}{c}\text { High-pressure } \\
\text { treatment (min) }\end{array}$ & $M_{\mathrm{w}}$ & $M_{\mathrm{n}}$ & $\begin{array}{c}\text { PDI } \\
\left(M_{\mathrm{w}} / M_{\mathrm{n}}\right)\end{array}$ \\
\hline 0 & 73400 & 18800 & 3.9 \\
30 & 11300 & 1100 & 10.9 \\
\hline
\end{tabular}

GPC measurements in a mixture of chloroform/1,1,1,3,3,3-hexafluoro-2propanol $(98 / 2 \mathrm{vol} \%)$ mobile phase at $40^{\circ} \mathrm{C}$ calibrated with polystyrene standards.

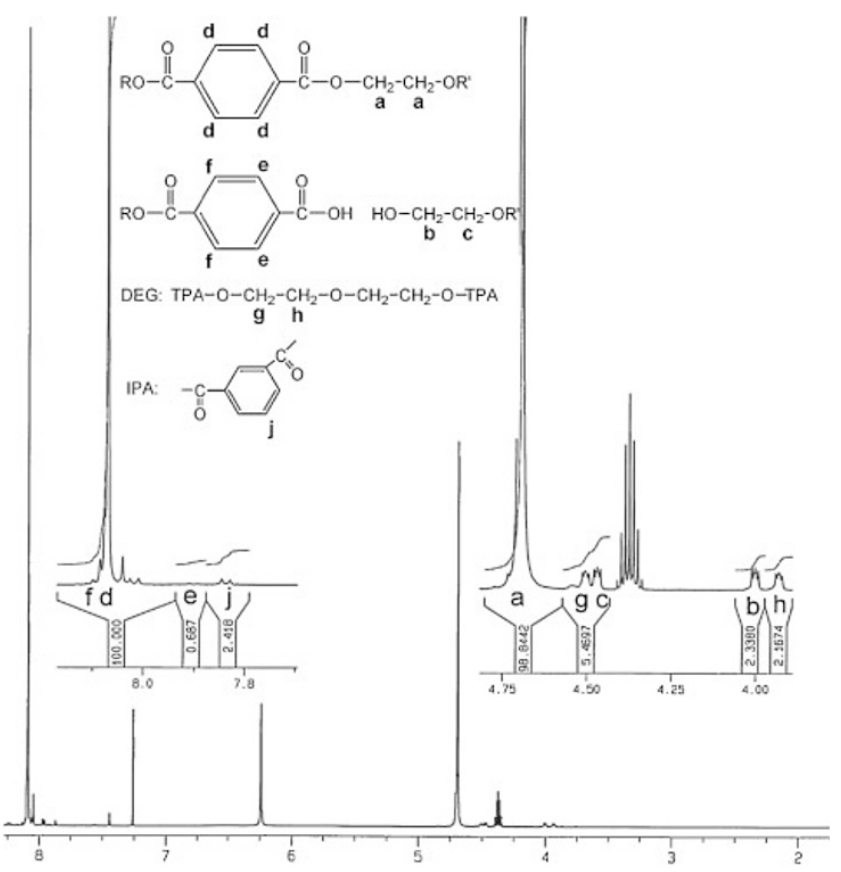

Figure 6. The ${ }^{1} \mathrm{H}$ NMR spectra of the sample during 30 -min high-pressure steam treatment.

presence of low-molecule-weight fractions and the absence of a solvent and standard substance. The degree of decomposition of PET was confirmed from the number average molecular weight and number of hydroxyls and acids based on the ratio of the integral value of ${ }^{1} \mathrm{H}$ NMR (Figures 7 and 8). From Figure 6 , the $M_{\mathrm{n}}$ of the samples was calculated as follows:

$$
\begin{aligned}
M_{\mathrm{n}} & =\frac{N\left(60\left(S_{\mathrm{a}} / 4+S_{\mathrm{b}} / 2\right)+132\left(S_{\mathrm{d}} / 4+S_{\mathrm{e}} / 2\right)\right)}{S_{\mathrm{a}} / 4+S_{\mathrm{b}} / 2+S_{\mathrm{d}} / 4+S_{\mathrm{e}} / 2} \\
N & =\frac{S_{\mathrm{a}} / 4+S_{\mathrm{b}} / 2+S_{\mathrm{d}} / 4+S_{\mathrm{e}} / 2}{1 / 2\left(S_{\mathrm{b}} / 2+S_{\mathrm{e}} / 2\right)}
\end{aligned}
$$

where $N$ is the number of repeating monomer units in one molecule, $S_{\mathrm{a}}$ and $S_{\mathrm{b}}$ are the methylene proton integrals of backbone (a: $4.70 \mathrm{ppm}, 4 \mathrm{H}, \mathrm{s}, \mathrm{OCH}_{2}$ ) and end-group (b: $4.00 \mathrm{ppm}, 2 \mathrm{H}$, dd, $\mathrm{HOCH}_{2}$ ) in EG units, $S_{\mathrm{d}}$ and $S_{\mathrm{e}}$ are aromatic proton integrals of backbone (d: $8.09 \mathrm{ppm}, 4 \mathrm{H}$, s, aromatic $H$ ) and end-group (e: $7.97 \mathrm{ppm}, 2 \mathrm{H}$, dd, aromatic $H$ ) in TPA units, respectively. The amounts of diethylene glycol (DEG) and isophthalic acid (IPA) in PET bottle were very little so they were not included in the calculations. Figure 7 shows that $M_{\mathrm{n}}$ decreased as the high-pressure steam treatment time increased, and the same tendency was observed in GPC analysis. Figure 8

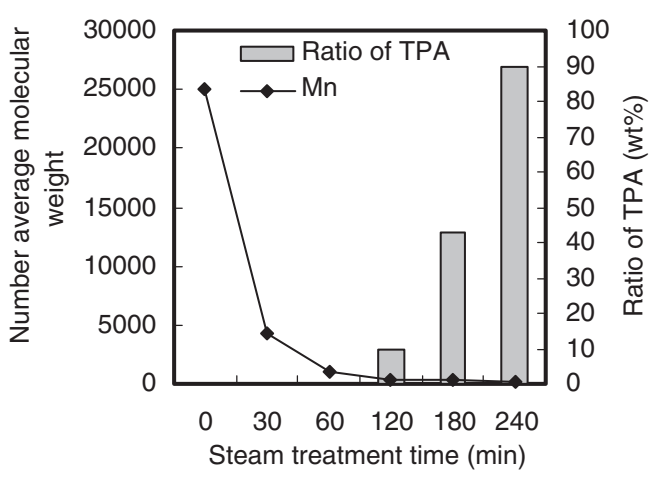

Figure 7. Variation in number average molecular weight by ${ }^{1} \mathrm{H}$ NMR and ratio of TPA with high-pressure steam treatment time.

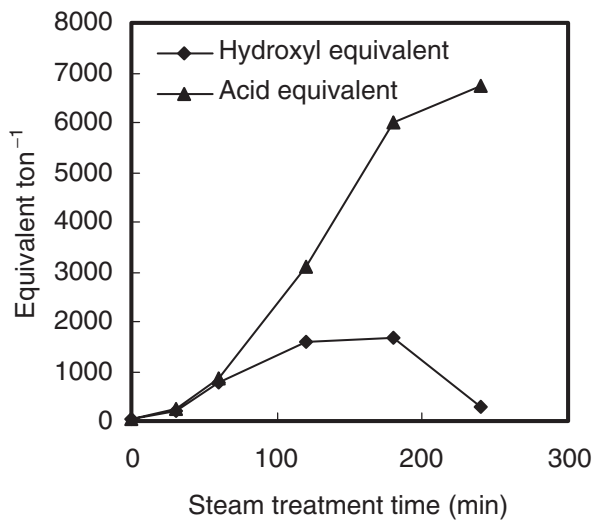

Figure 8. Variation in the quantities of end groups of PET with high-pressure steam treatment time.

indicates that the number of hydroxyls and carboxylic acids increase at the steam treatment time exceeding $180 \mathrm{~min}$. During the high-pressure steam treatment, PET was gradually reduced to oligomer, and then further reduced to monomer. The samples treated for 120,180 , and $240 \mathrm{~min}$ were centrifuged because they did not completely melt in the solvent. According to the ${ }^{1} \mathrm{H}$ NMR spectra: $\delta 8.00(\mathrm{~d}, 4 \mathrm{H}$, aromatic $H), 3.35(\mathrm{~s}, 2 \mathrm{H}$, $\mathrm{COOH}$ ), the sediment after centrifugation was found to TPA.

When the sample was processed for more than $180 \mathrm{~min}$, the number of hydroxyls decreased because ethylene glycol in the monomer dissolved in water. Liquid collected after the 240min treatment contained ethylene glycol according to the ${ }^{1} \mathrm{H}$ NMR spectra: $\delta 3.50$ (s, 4H, $\mathrm{HOCH}_{2}$ ).

When PET completely decomposed to monomers, maximum yields of TPA and EG were $67 \%$ and $31 \%$, respectively, and the remaining $2 \%$ was DEG and IPA according to the ${ }^{1} \mathrm{H}$ NMR of raw material (PET bottles). When TPA and EG monomers from the decomposed PET were further treated for $240 \mathrm{~min}, 64 \% \mathrm{TPA}$ and $29 \% \mathrm{EG}$ monomers were retrieved, and corresponded to $96 \%$ and $94 \%$ of the maximum yields, respectively.

PET effectively decomposes by high-pressure steam treatment into fine particles. Molecular weights, $T_{\mathrm{g}}, T_{\mathrm{c}}$, and $T_{\mathrm{m}}$ of the decomposed fine particles substantially decreased with high 
degree of crystallization during steam treatment time. Based on the above, a molecular design system for recycling PET was developed. And differs from the supercritical reaction system in which the reaction is so fast that the monomer might undergo as decomposition. In this technology, PET decomposes into a monomer, a reusable raw material in decomposed PET such as the oligomer is a compound consisting of hydroxyl groups and carboxylic acid, and undergoes chemical reaction with polymerization agents and is remolded into new products. This method is applicable not only to the ester bond but also to condensation reactions such as those involving the urea bond and urethane bond. ${ }^{14}$

The high-pressure steam treatment is a single-step process, while present recycling methods involve multiple steps such as washing, crushing, and drying. This method in combination with the present collection system for used PET bottles showed be economically viable.

\section{CONCLUSION}

The molecular weight of PET gradually decreases to that of an oligomer with the high-pressure steam treatment time without crushing or using acidic or alkaline catalysts and solvents, and then is further reduced to a monomer.

Acknowledgment. The authors thank Hirotaka Murate, Assoc. Prof. Kouzou Yoshitani, Gifu University, for their comments and support in this study. They thank John Tawiah Adomako (Ph D.) for proofreading this report.

Received: December 13, 2007

Accepted: February 9, 2008

Published: April 9, 2008

\section{REFERENCES}

1. N. Kusakawa, Plastic Age, 44, 156 (1998).

2. H. Miwa, Kogyozairyo, 46, 23 (1998).

3. H. Grunschke, W. Hammerschick, and B. Naucheim, U. S. Patent 3 403115 (1968).

4. S. Baliga and W. T. Wong, J. Polym. Sci., Part A: Polym. Chem., 27, 2071 (1989).

5. T. Yoshioka and A. Okumura, Ryosan to kogyo, 47, 21 (1994).

6. "Science and Technology of Supercritical Fluids," S. Saito, Ed., Sankyo Business, (1996).

7. T. Sako, T. Sugeta, K. Otake, N. Nakazawa, M. Sato, K. Namiki, and M. Tsugumi, J. Chem. Eng. Jpn., 30, 342 (1997).

8. T. Sako, T. Sugeta, K. Otake, Y. Takebayashi, C. Kamizawa, M. Tsugumi, and M. Hongo, Kobunshi Ronbunshu, 55, 11 (1998).

9. T. Sako, T. Sugeta, and I. Okajima, Shigen Kankyo Taisaku, 34, 1147 (1998).

10. Y. Nagase, Koatsuryoku no Kagaku to Gijyutsu, 12, 217 (2002).

11. M. Goto, M. Sakai, and T. Hirose, J. Mater. Sci., 41, 1509 (2006).

12. W. B. Holzapfel, J. Chem. Phy, 50, 4424 (1969).

13. K. Todheid, in "Water-A Comprehensive Treatise" vol. 1, F. Franks, Ed., Plenum Press, New York, 1972, p 463.

14. A. Noritake, M. Shigematsu, T. Tsuji, and M. Tanahashi, Trans. Mater. Res. Soc. Jpn., 32, 705 (2007). 